\section{Associação entre carência social e causas de morte entre idosos residentes no Município de Recife, Pernambuco, Brasil}

\author{
Association between social deprivation and causes \\ of mortality among elderly residents in the city of \\ Recife, Pernambuco State, Brazil
}

\author{
${ }_{1}$ Universidade Federal de \\ Pernambuco, Recife, Brasil \\ Correspondência \\ V. L. Silva \\ Departamento de Medicina \\ Social, Universidade Federal \\ de Pernambuco. \\ Av. Professor Moraes Rego s $/ n$ \\ Cidade Universitária, \\ Recife, $P E$ \\ 50000-000, Brasil. \\ vlsilva@pop.com.br
}

\begin{abstract}
This paper aims to analyze mortality among elderly residents in the city of Recife, Pernambuco State, Brazil, and its association with social deprivation (hardship) in the year 2000. An ecological study was performed, and 94 neighborhoods and 5 social strata were analyzed. The independent variable consisted of a composite social deprivation indicator, obtained for each neighborhood and calculated through a scoring technique based on census variables: water supply, sewerage, illiteracy, and head-of-household's years of schooling and income. The dependent variables were: mortality rate in individuals $>60$ years of age and cause-specific mortality rates. The association was calculated by means of the Pearson correlation coefficient, linear regression, and mortality odds between social deprivation strata formed by grouping of neighborhoods according to the indicator's quintiles. The data show a statistically significant positive correlation between social deprivation and mortality in the elderly from pneumonia, protein-energy malnutrition, tuberculosis, diarrhea/gastroenteritis, and traffic accidents, and a negative correlation with deaths from bronchopulmonary and breast cancers.
\end{abstract}

Mortality; Older; Socioeconomic Inequalities
Vanessa de Lima Silva 1

Márcia Carréra Campos Leal 1

Jacira Guiro Marino 1

Ana Paula de Oliveira Marques 1

\section{Introdução}

O envelhecimento populacional é um fenômeno mundial. No século XX observou-se importante aumento da proporção de idosos, crescimento este mais acentuado nos países em desenvolvimento. No Brasil o processo encontra-se em suas etapas iniciais, a participação de idosos no total da população passou de 4\% em 1940 para 8,6\% em 2000, correspondendo a 14.536.029 idosos (Censo Demográfico 2000. Instituto Brasileiro de Geografia e Estatística - IBGE; http://www.ibge. gov.br). Segundo Carvalho \& Garcia 1, o envelhecimento da população brasileira se dará em ritmo maior do que o ocorrido nos países de primeiro mundo, em virtude da acentuada queda de fecundidade observada no país.

A situação demográfica e epidemiológica da população brasileira configura-se num mosaico extremamente complexo, em virtude da distribuição desigual dos riscos e agravos nos diversos grupos populacionais, expressa nas diferenças observadas em taxas e coeficientes das diversas regiões do país, entre micro-regiões do mesmo estado, ou nas diferenças encontradas entre zonas de informação da mesma cidade 2 .

Segundo Castellanos 3, a mortalidade é influenciada por processos de caráter contraditório, dentre os quais podem-se destacar: as iniqüidades sociais e desigualdade nas condições de vida; o processo de urbanização; mudanças na estrutura de idade da população, na composição 
da força de trabalho e no mercado de trabalho, no nível de educação da população e mudanças na organização dos serviços de saúde.

A problemática da influência da condição de vida na situação de saúde é alvo de estudos em todo o mundo. O registro de diferenciais de mortalidade entre classes sociais segundo variáveis sócio-econômicas tem sido feito em países com distintos níveis de desenvolvimento, tais como Finlândia, Suécia, Noruega, Dinamarca, Inglaterra e Itália 4,5, Estados Unidos e Alemanha 6, Grã-Betanha e Japão 7, Coréia 8, Austrália 9, Argentina 10 e Brasil 11,12,13,14,15.

Quando se trata de estudos relacionados à situação de saúde de idosos, há uma significativa redução do número de pesquisas, concentradas em sua maioria em países da Europa. Dentre estes, destacam-se os estudos de Huisman et al. 16,17 que analisaram a associação entre a mortalidade de idosos e a condição sócio-econômica dos mesmos, representada por nível de escolaridade e condição de habitação em grupos de países europeus. Na América Latina, Noronha \& Andrade 18 realizaram estudo sobre as desigualdades sociais em saúde entre idosos das cidades de Buenos Aires, São Paulo, Santiago, Havana, Cidade do México e Montevidéu.

No Brasil, são raras as referências de pesquisas com esta temática, podendo-se citar os trabalhos de Lima-Costa et al. 19,20. Baseados em dados da Pesquisa Nacional por Amostra de Domicílios (PNAD), as autoras avaliaram a associação entre indicadores de saúde, de mobilidade física e uso de serviços de saúde, referidos por idosos brasileiros e sua situação sócio-econômica, caracterizada pela variável renda.

Em relação à associação entre mortalidade da população idosa e condição sócio-econômica, não foram encontrados estudos realizados no Brasil. No entanto, há uma significativa produção de pesquisas brasileiras que buscam tal associação com a mortalidade do adulto. Estes trabalhos foram realizados nas cidades de São Paulo 21, Salvador 11, Rio de Janeiro 12 e Belo Horizonte 22 .

Nestes estudos a condição sócio-econômica foi mensurada de acordo com indicadores e metodologias diversas, esta é uma importante limitação quando se deseja comparações interregionais. No entanto os autores chegaram a resultados em comum. É consenso entre as pesquisas que os indicadores de mortalidade variam de acordo com diferenciais sociais e econômicos, a maioria destes indicadores apresenta valores mais elevados em áreas de piores condições sócio-ambientais.

Huisman et al. 16 estudaram 11 populações do norte, sul e países centrais da Europa. Foi rea- lizado um estudo comparativo entre mortalidade em idosos e adultos jovens, a pesquisa forneceu evidências de que as desigualdades sócio-econômicas persistem na mortalidade entre populações idosas da Europa, um grande número de mortes adicionais que ocorre entre os grupos sócio-econômicos mais baixos é um importante problema de saúde pública.

As três causas mais freqüentes de óbito entre idosos brasileiros são doenças do aparelho circulatório, neoplasias e doenças do aparelho respiratório tanto em 1980 e 1991 quanto em 2000. Contudo, há sinais de mudança. As taxas de mortalidade por doenças do aparelho circulatório apresentam um acentuado e constante declínio, ao passo que as taxas de mortalidade por neoplasias e doenças do aparelho respiratório aumentaram gradativamente. As mortes por causas externas, que não tinham um papel importante no total dos óbitos da população idosa, estão aumentando seu peso relativo 23,24.

Em estudo realizado em São Paulo, Drumond Jr. \& Barros 21 relatam diferenças sociais importantes nos coeficientes de morte em adultos paulistanos por doenças infecciosas (tais como AIDS, pneumonias, tuberculose), por doenças crônicas não transmissíveis (doenças cardiovasculares e alguns cânceres, entre outras) e por causas externas. Os autores referem que com poucas exceções os coeficientes são maiores nas regiões de piores condições sócio-ambientais.

A associação entre a prevalência de doenças crônicas e a condição social foi relatada por Barros et al. 25 , os autores referem que o segmento de menor escolaridade da população adulta brasileira apresentou $62 \%$ a mais de prevalência de doenças crônicas, se comparado aos de maior nível.

Santos \& Noronha 13 analisaram as relações dos padrões espaciais de mortalidade dos 153 bairros da cidade do Rio de Janeiro com o perfil sócio-econômico dos bairros e nas microáreas sócio-economicamente homogêneas que compõem o município. O padrão espacial dos estratos sócio-econômicos retratou as diversas condições de vida dos cariocas implicando padrões de mortalidade específicos. Os estratos sócio-economicamente mais favorecidos apresentaram um perfil de baixa mortalidade para todas as causas.

O estudo intra-urbano dos padrões demográficos e epidemiológicos pode contribuir com a formulação de políticas e planejamento das ações de saúde com observações mais precisas das necessidades de saúde das populações.

Considerando a importância do tema, este trabalho se propõe a analisar a associação entre carência social e causas de morte da população 
idosa residente na cidade do Recife, Pernambuco, Brasil.

\section{Método}

Trata-se de um estudo ecológico, de agregados populacionais, tendo como área de estudo o Município do Recife, capital do Estado de Pernambuco, totalmente urbano, possui 94 bairros e uma população de 1.422.905 habitantes, destes 133.532 habitantes são idosos.

A unidade de análise correspondeu aos 94 bairros da cidade e 5 estratos de carência social, onde foi analisado o universo de óbitos da população idosa (60 anos e mais), residente no Recife. De um total de 5.594 óbitos foram excluídos 12 que apresentavam bairro ignorado, restando 5.582 óbitos de idosos, os quais foram utilizados neste estudo. O período de referência para esta pesquisa é o ano 2000, tanto para os dados referentes à mortalidade, como para os dados censitários referentes à população e condição sócioeconômica.

A coleta de dados foi realizada através do Sistema de Informações sobre Mortalidade (SIM) da Prefeitura do Recife e do Censo Demográfico de 2000 do IBGE. No processamento e análise de dados foram utilizados os programas Tabwin 32 (Departamento de Informática do SUS DATASUS; http://www.datasus.gov.br), ArcView 3.2 (Environmental Systems Research Institute Inc., http://www.esri.com/software/arcview/) e SPSS 13 (SPSS Inc., Chicago, Estados Unidos).

A situação sócio-econômica da cidade do Recife foi mensurada através de um indicador composto de carência social (ICS), este indicador consistiu a variável independente do estudo e foi construído através da técnica de formação de escores, baseado em estudos anteriores $26,27,28$. Segundo a técnica, a partir das variáveis eleitas, efetuou-se a ordenação dos bairros segundo valor de cada variável isoladamente, estabelecendo-se então o escore (ES) de cada bairro, de forma que foram identificados o menor valor encontrado $\left(\mathrm{V}_{\text {min }}\right)$ e o maior valor $\left(\mathrm{V}_{\max }\right)$ de cada variável para cada bairro. Em seguida foi feita uma interpolação para obtenção dos escores para cada bairro usando-se a relação $\mathrm{ES}_{\mathrm{ij}}=\left(\mathrm{V}_{\mathrm{obs}}-\mathrm{V}_{\min }\right) /\left(\mathrm{V}_{\max }\right.$ $\left.-\mathrm{V}_{\text {min }}\right)$, que possibilitou colocar todas as variáveis dentro de uma mesma escala. O ICS foi então obtido para cada bairro como média aritmética simples dos escores obtidos em cada bairro (i) para cada variável (j), multiplicada por 100, para redução do número de casas decimais, ou seja, $\mathrm{ICS}=100 \times \sum \mathrm{ES}_{\mathrm{ij}} / \mathrm{n}$, onde n é o número de variáveis eleitas. Os bairros foram agrupados por quintil do ICS, constituindo-se então em estra- tos homogêneos de carência social da cidade do Recife.

As variáveis censitárias utilizadas para a construção do indicador composto foram baseadas no estudo de Silva ${ }^{15}$, sendo: percentual de domicílios com abastecimento de água não canalizada, percentual de domicílios sem instalação sanitária, percentual da população de 10 a 14 anos analfabeta, percentual de responsável pelo domicílio com 3 anos ou menos de estudo e percentual de chefe de domicílio com renda mensal menor ou igual a 2 salários mínimos.

Como variáveis dependentes foram avaliados neste estudo: coeficientes de mortalidade padronizados por idade e sexo, de acordo com a metodologia proposta por Kirkwood 29, em maiores de 60 anos e coeficientes de mortalidade específicos para idosos por causa básica de óbito, calculados para cada bairro e estratos de carência social.

As causas de morte analisadas neste estudo apresentam os seguintes códigos de acordo com a Classificação Estatística Internacional de Doenças e Problemas Relacionados à Saúde 10a Revisão (CID-10) 30: tuberculose em vias respiratórias (A16); diarréia e gastroenterite (A09); outras septicemias (A41); neoplasia maligna de brônquios e pulmões (C34); neoplasia maligna de próstata (C61); neoplasia maligna da mama (C50); diabetes mellitus (E14); desnutrição protéico-calórica (E46); infarto agudo do miocárdio (I21); acidente vascular cerebral (I64); doença isquêmica crônica do coração (I25); transtornos respiratórios (J98); pneumonia (J18); doença pulmonar obstrutiva crônica (J44); outras doenças do fígado (K76); fibrose e cirrose hepáticas (K74); transtornos vasculares do intestino (K55); quedas (W01, 06, 07, 09.3, 10, 17, 18, 19); acidente de transporte (V09, 49, 89) e agressões (X95, Y 00, 04).

Os dados diretos do SIM apresentam, na atualidade, grande potencial de uso para os estudos de mortes, devido ao acompanhamento sistemático da ocorrência do evento. Com a considerável redução de subnotificação, os esforços se concentram na melhoria da qualidade do preenchimento das declarações de óbitos. Nesse sentido observa-se o crescente declínio de campos não preenchidos e os com alternativa ignorado ${ }^{31}$.

A associação entre mortalidade em idosos e carência social foi mensurada através do coeficiente de correlação de Pearson entre o indicador composto de carência social e os indicadores de mortalidade em idosos.

Também foi realizada a regressão linear simples para obtenção do modelo explicativo da mortalidade em idosos a partir do ICS, ou seja, foi ajustada uma reta que expressa o comportamento conjunto de cada coeficiente de mortalidade em idosos e do indicador de carência social 
do bairro em que ele reside, sendo o indicador de carência social a variável independente e os coeficientes de mortalidade em idosos e por causa básica de morte, padronizados por faixa etária e sexo as variáveis dependentes, ou seja, aquelas que serão previstas a partir da informação da variável independente. Em todas as análises foi utilizado o nível de significância de 5\%.

A associação foi ainda mensurada através da razão de mortalidade entre os estratos de carência social, foi calculada a razão dos indicadores de cada estrato e aquele do estrato um, de baixa carência social, considerado como referência.

Quanto às considerações éticas, os dados relativos à mortalidade foram fornecidos pela Secretaria Municipal de Saúde mediante termo de cessão e compromisso de que será resguardada a confidencialidade da identificação dos óbitos registrados. Este projeto foi aprovado pelo Comitê de Ética em Pesquisas em Seres Humanos,

Figura 1

Distribuição de bairros segundo estratos de carência social. Recife, Pernambuco, Brasil, 2000.

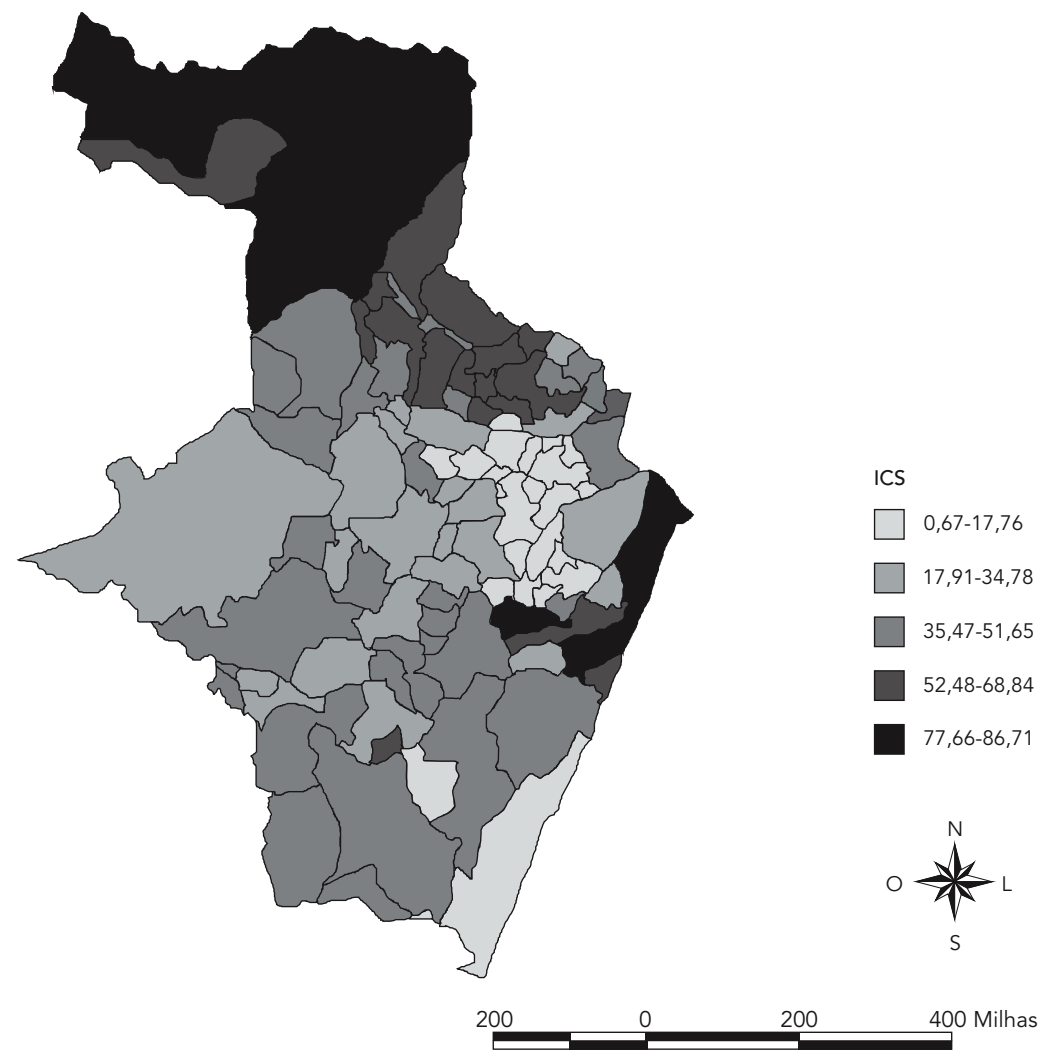

ICS: indicador composto de carência social. do Centro de Ciências da Saúde da Universidade Federal de Pernambuco (Ofício no. 122/2006CEP/CCS), estando de acordo com a Resolução $n^{\circ} .196 / 96$.

\section{Resultados}

A cidade do Recife apresenta-se com um padrão de significativa desigualdade social, a distribuição do ICS entre os bairros mostrou-se heterogênea, com variação de 0,67 para Graças, bairro menos carente da cidade, até 86,71 para o bairro Recife, de muito alta carência social (Figura 1).

O coeficiente de mortalidade padronizado por idade e sexo da população recifense acima de 60 anos apresenta valor médio entre os bairros da cidade de 42,5 óbitos para cada mil habitantes (Tabela 1). Neste estudo não foi encontrada associação estatisticamente significativa da mortalidade de recifenses maiores de 60 anos e a carência social da população, no entanto, ao se estratificar os óbitos analisados por causa básica da morte, foram encontradas associações.

As doenças do aparelho circulatório são as maiores causas de morte entre idosos residentes no Recife, cujo coeficiente de mortalidade médio entre os bairros da cidade é de 188,7 óbitos/10 mil habitantes. O segundo grupo de causas de morte entre a população é representado pelas neoplasias, com média de 66,9 óbitos/10 mil habitantes. A terceira causa de óbito são as doenças do aparelho respiratório, seguidas das doenças endócrinas, nutricionais e metabólicas, doenças do aparelho digestivo, doenças infecciosas e parasitárias e causas externas (Tabela 1).

No presente estudo não foi encontrada correlação estatisticamente significativa entre a carência social e a mortalidade de idosos por doenças do aparelho circulatório, apesar da literatura especializada referir tal associação. No entanto, ao se analisar a razão de prevalência entre os estratos de carência social, encontrou-se aumento do risco de morte com o aumento da carência. Este padrão também foi observado para as causas específicas infarto agudo do miocárdio e acidente vascular cerebral (Tabela 2).

A mortalidade por neoplasias apresentou correlação negativa com a carência social, inferindo-se aumento de mortes nos grupos mais favorecidos socialmente (Tabela 3). A neoplasia maligna de brônquios e pulmões apresentou a mesma tendência, sendo mais forte em relação às demais neoplasias, com coeficiente de correlação de Pearson -0,31 e modelo de regressão linear explicando 9,8\% da associação. A análise da razão de mortalidade entre os estratos de carência social para esta causa, demonstrou dimi- 
Estatística descritiva de coeficientes de mortalidade em idosos padronizados por faixa etária e sexo, segundo causa básica e causas mais freqüentes de morte. Recife, Pernambuco, Brasil, 2000

\begin{tabular}{|c|c|c|c|c|c|c|c|}
\hline Variável & Média & $\begin{array}{l}\text { Desvio- } \\
\text { padrão }\end{array}$ & $\begin{array}{l}\text { Valor } \\
\text { mínimo }\end{array}$ & $\begin{array}{c}\text { Percentil } \\
25\end{array}$ & Mediana & $\begin{array}{c}\text { Percentil } \\
75\end{array}$ & $\begin{array}{l}\text { Valor } \\
\text { máximo }\end{array}$ \\
\hline Coeficiente de mortalidade em idoso * & 42,5 & 17,8 & 0 & 33,5 & 42,3 & 48,9 & 119,2 \\
\hline \multicolumn{8}{|l|}{ Causa básica ** } \\
\hline Doenças do aparelho circulatório & 188,7 & 111,8 & 0 & 118,0 & 178,4 & 232,3 & 708,9 \\
\hline Infarto agudo do miocárdio & 52,5 & 38,2 & 0 & 30,8 & 46,8 & 66,1 & 225,4 \\
\hline Acidente vascular cerebral & 20,3 & 28,0 & 0 & 0 & 15,5 & 26,9 & 225,4 \\
\hline Doença isquêmica crônica do coração & 13,7 & 14,4 & 0 & 0 & 12,3 & 20,9 & 60,3 \\
\hline Neoplasias & 66,9 & 57,5 & 0 & 34,6 & 64,3 & 80,6 & 457,3 \\
\hline Maligna de brônquio/pulmões & 7,7 & 12,4 & 0 & 0 & 0 & 11,6 & 69,3 \\
\hline Maligna da próstata & 7,8 & 14,8 & 0 & 0 & 1,3 & 12,5 & 110,9 \\
\hline Maligna da mama & 5,6 & 11,4 & 0 & 0 & 0 & 7,4 & 77,4 \\
\hline Doenças do aparelho respiratório & 57,3 & 46,4 & 0 & 31,1 & 52,4 & 73,8 & 353,8 \\
\hline Transtornos respiratórios & 21,1 & 17,9 & 0 & 0 & 18,2 & 32,0 & 66,6 \\
\hline Pneumonia & 14,9 & 37,5 & 0 & 0 & 8,9 & 19,7 & 353,8 \\
\hline Doença pulmonar obstrutiva crônica & 8,1 & 13,4 & 0 & 0 & 0 & 14,5 & 69,4 \\
\hline Doenças endócrinas, nutricionais e metabólicas & 38,8 & 35,1 & 0 & 16,3 & 31,7 & 54,6 & 222,5 \\
\hline Diabetes mellitus & 25,5 & 25,1 & 0 & 0 & 19,9 & 40,3 & 135,9 \\
\hline Desnutrição protéico-calórica & 7,5 & 12,3 & 0 & 0 & 1,2 & 12,7 & 71,1 \\
\hline Doenças do aparelho digestivo & 21,1 & 19,8 & 0 & 0 & 17,8 & 33,6 & 101,1 \\
\hline Outras doenças do fígado & 2,8 & 5,4 & 0 & 0 & 0 & 3,0 & 25,0 \\
\hline Fibrose e cirrose hepáticas & 2,6 & 5,5 & 0 & 0 & 0 & 3,0 & 29,9 \\
\hline Transtornos vasculares do intestino & 1,4 & 4,0 & 0 & 0 & 0 & 0 & 30,8 \\
\hline Doenças infecciosas e parasitárias & 19,2 & 30,5 & 0 & 0 & 13,7 & 25,4 & 234,3 \\
\hline Tuberculose nas vias respiratórias & 5,3 & 16,6 & 0 & 0 & 0 & 7,6 & 150,6 \\
\hline Diarréia e gastroenterite & 2,6 & 5,2 & 0 & 0 & 0 & 3,4 & 31,3 \\
\hline Outras septicemias & 2,7 & 7,6 & 0 & 0 & 0 & 2,9 & 63,4 \\
\hline Causas externas & 10,4 & 17,9 & 0 & 0 & 9,1 & 13,8 & 150,6 \\
\hline Quedas & 2,7 & 5,7 & 0 & 0 & 0 & 3,2 & 32,2 \\
\hline Acidente de transporte & 3,4 & 15,8 & 0 & 0 & 0 & 2,0 & 150,6 \\
\hline Agressões & 1,4 & 4,9 & 0 & 0 & 0 & 0 & 29,9 \\
\hline
\end{tabular}

* Coeficiente na base de mil habitantes;

** Coeficiente na base de 10 mil habitantes.

nuição gradativa do risco de morte à medida do aumento da carência.

Tendência semelhante apresentou a neoplasia maligna de mama. Foi encontrada associação negativa e razão de mortalidade indicando diminuição do risco de morte com o aumento da carência social.

Quanto ao grupo de doenças do aparelho respiratório, foi encontrada correlação positiva com a carência social. O coeficiente de mortalidade por pneumonia apresentou correlação positiva e razão de mortalidade indicando aumento gradativo do risco de morte em direção aos estratos mais carentes. Idosos residentes no estrato de muito alta carência social apresentam risco de morte por pneumonia 3,9 vezes maior em comparação a idosos residentes no estrato de baixa carência (Tabela 2).

A mortalidade de idosos por desnutrição protéico-calórica apresenta risco crescente com o aumento da carência social. Idosos residentes nos bairros do estrato de muito alta carência social têm risco 9,6 vezes maior de morrer por desnutrição do que idosos residentes nos bairros recifenses de baixa carência social (Tabela 2).

Dentre as doenças infecciosas e parasitárias, a tuberculose de vias respiratórias é a maior causa de morte entre idosos recifenses. A mortalidade apresenta correlação positiva com a carência social $(r=0,32)$ e modelo de regressão linear expli- 
Razão de mortalidade em idosos entre estratos de carência social, segundo grupo de causa e causas mais freqüentes de morte. Recife, Pernambuco, Brasil, 2000

\begin{tabular}{|c|c|c|c|c|}
\hline \multirow[t]{2}{*}{ Indicador * } & \multicolumn{4}{|c|}{ Razão de mortalidade entre estratos de carência social } \\
\hline & II/I & III/I & IV/I & $\mathrm{V} / \mathrm{I}$ \\
\hline \multicolumn{5}{|l|}{ Coeficiente de mortalidade } \\
\hline Por doenças do aparelho circulatório & 1,5 & 1,5 & 1,8 & 1,3 \\
\hline Por infarto agudo do miocárdio & 1,6 & 1,6 & 1,6 & 1,0 \\
\hline Por acidente vascular cerebral & 1,5 & 1,5 & 1,8 & 0,7 \\
\hline Por doença isquêmica crônica do coração & 0,8 & 1,1 & 1,0 & 0,0 \\
\hline \multicolumn{5}{|l|}{ Por neoplasias } \\
\hline Por neoplasia maligna de brônquios/pulmões & 1,0 & 0,8 & 0,8 & 0,2 \\
\hline Por neoplasia maligna da próstata & 0,6 & 0,6 & 0,5 & 0,0 \\
\hline Por neoplasia maligna da mama & 1,3 & 1,1 & 0,5 & 0,0 \\
\hline Por doenças do aparelho respiratório & 1,3 & 0,8 & 0,3 & 0,0 \\
\hline \multicolumn{5}{|l|}{ Por transtornos respiratórios } \\
\hline Por pneumonia & 1,3 & 1,1 & 1,2 & 1,3 \\
\hline Por doença pulmonar obstrutiva crônica & 0,9 & 0,8 & 0,9 & 0,0 \\
\hline Por doenças endócrinas, nutricionais e metabólicas & 2,0 & 2,0 & 2,4 & 3,9 \\
\hline Por diabetes mellitus & 1,6 & 1,1 & 1,5 & 1,0 \\
\hline \multicolumn{5}{|l|}{ Por desnutrição protéico-calórica } \\
\hline Por doenças do aparelho digestivo & 1,6 & 1,7 & 2,1 & 1,9 \\
\hline Por outras doenças do fígado & 1,9 & 2,1 & 2,9 & 0,6 \\
\hline Por fibrose e cirrose hepáticas & 2,0 & 2,8 & 2,4 & 9,6 \\
\hline \multicolumn{5}{|l|}{ Por transtornos vasculares do intestino } \\
\hline Por doenças infecciosas e parasitárias & 1,4 & 1,8 & 1,4 & 1,2 \\
\hline Por tuberculose nas vias respiratórias & 4,6 & 3,6 & 4,7 & 8,5 \\
\hline Por diarréia e gastroenterite & 1,3 & 1,8 & 1,7 & 0,0 \\
\hline Por outras septicemias & 1,0 & 1,9 & 2,6 & 0,0 \\
\hline \multicolumn{5}{|l|}{ Por causas externas } \\
\hline Por quedas & 1,5 & 1,6 & 1,9 & 1,6 \\
\hline Por acidente de transporte & 2,3 & 4,4 & 4,6 & 5,2 \\
\hline Por agressões & 4,1 & 3,1 & 6,5 & 11,2 \\
\hline Por doenças do aparelho circulatório & 0,8 & 0,5 & 0,6 & 0,0 \\
\hline \multicolumn{5}{|l|}{ Por infarto agudo do miocárdio } \\
\hline Por acidente vascular cerebral & 1,1 & 0,9 & 0,9 & 2,3 \\
\hline Por doença isquêmica crônica do coração & 1,1 & 0,7 & 0,5 & 0,0 \\
\hline Por neoplasias & 1,7 & 0,8 & 1,7 & 2,9 \\
\hline Por neoplasia maligna de brônquios/pulmões & 0,8 & 1,7 & 0,8 & 0,0 \\
\hline
\end{tabular}

* Coeficientes padronizados por faixa etária e sexo.

cando 10,1\% da associação (Tabela 3). Na análise da razão de mortalidade encontrou-se aumento gradativo do risco de morte concomitante ao aumento da carência social.

A mortalidade por diarréia e gastroenterite também apresentou correlação positiva com a carência social. A razão de mortalidade apresentou tendência crescente com o aumento da carência. À semelhança dos dados de tuberculose, a diarréia tem forte associação com as condições sociais e econômicas dos grupos populacionais.
As causas externas constituem a sétima causa de morte entre os idosos recifenses, na análise de associação com a carência social, foi encontrada correlação positiva estatisticamente significativa $(r=0,22)$, podendo-se inferir que idosos residentes em bairros de alta carência social estão mais expostos a injúrias decorrentes do meio em comparação aos idosos residentes em áreas de melhor condição de vida. As causas externas de mortalidade mais prevalentes entre os idosos do Recife são as quedas, seguidas de acidentes de 
Coeficientes de correlação de Pearson e resultados da regressão linear entre a carência social e a mortalidade em idosos padronizada por faixa etária e sexo, segundo grupo de causa e causas mais freqüentes de morte. Recife, Pernambuco, Brasil, 2000

\begin{tabular}{|c|c|c|c|}
\hline \multirow[t]{2}{*}{ Indicador } & \multirow{2}{*}{$\begin{array}{l}\text { Coeficiente } \\
\text { correlação }\end{array}$} & \multicolumn{2}{|c|}{ Regressão linear } \\
\hline & & Equação da reta & $\begin{array}{c}\text { Coeficiente de } \\
\text { determinação (\%) }\end{array}$ \\
\hline \multicolumn{4}{|l|}{ Coeficiente de mortalidade } \\
\hline Em idoso & 0,12 & $Y=38,46+0,11 * I C S$ & 1,4 \\
\hline Por doenças do aparelho circulatório & 0,11 & $Y=166,30+0,62 * I C S$ & 1,2 \\
\hline Por infarto agudo do miocárdio & $-0,00$ & $Y=52,49+6,89 * I C S$ & 0,0 \\
\hline Por acidente vascular cerebral & 0,06 & $Y=17,27+8,49 * I C S$ & 0,4 \\
\hline Por doença isquêmica crônica do coração & $-0,14$ & $Y=17,44-0,10 * I C S$ & 0,2 \\
\hline Por neoplasias & $-0,26$ ** & $Y=94,11-0,75 * I C S$ & 6,8 \\
\hline Por neoplasia maligna de brônquios/pulmões & $-0,31 * *$ & $Y=14,78-0,19 * I C S$ & 9,8 \\
\hline Por neoplasia maligna da próstata & $-0,16$ & $Y=12,13-0,12 * I C S$ & 2,6 \\
\hline Por neoplasia maligna da mama & $-0,27 * \star$ & $Y=11,21-0,15 * I C S$ & 7,4 \\
\hline Por doenças do aparelho respiratório & $0,23 * \star$ & $Y=37,61+0,54 * I C S$ & 5,4 \\
\hline Por transtornos respiratórios & $-0,14$ & $Y=25,52-0,12 * I C S$ & 1,9 \\
\hline Por pneumonia & $0,29 * \star$ & $Y=-5,07+0,56 \mathrm{ICS}$ & 8,7 \\
\hline Por doença pulmonar obstrutiva crônica & 0,01 & $Y=7,84+7,05 * I C S$ & 0,0 \\
\hline Por doenças endócrinas, nutricionais e metabólicas & 0,01 & $Y=38,26+1,44 * I C S$ & 0,0 \\
\hline Por diabetes mellitus & 0,07 & $Y=22,30+8,81 * I C S$ & 0,5 \\
\hline Por desnutrição protéico-calórica & 0,24 * & $Y=2,09+0,15 * I C S$ & 6,0 \\
\hline Por doenças do aparelho digestivo & $-0,01$ & $Y=20,82+6,34 * I C S$ & 0,0 \\
\hline Por outras doenças do fígado & 0,15 & $Y=1,29+4,14 * \operatorname{ICS}$ & 2,3 \\
\hline Por fibrose e cirrose hepáticas & 0,07 & $Y=1,85+2,10 * I C S$ & 0,6 \\
\hline Por transtornos vasculares do intestino & $-0,09$ & $Y=2,05-1,90 * I C S$ & 0,9 \\
\hline Por doenças infecciosas e parasitárias & 0,11 & $Y=13,34+0,16 * I C S$ & 1,1 \\
\hline Por tuberculose nas vias respiratórias & 0,32 ** & $Y=-4,23+0,26 * I C S$ & 10,1 \\
\hline Por diarréia e gastroenterite & 0,28 * & $Y=-1,74+7,29 * I C S$ & 7,7 \\
\hline Por outras septicemias & $-0,08$ & $Y=3,71-2,94 * I C S$ & 0,6 \\
\hline Por causas externas & 0,22 * & $Y=3,24+0,20 * I C S$ & 4,9 \\
\hline Por quedas & $-0,12$ & $Y=3,94-3,47 * I C S$ & 1,5 \\
\hline Por acidente de transporte & $0,27 * \star$ & $Y=-4,26+0,21 * I C S$ & 7,2 \\
\hline Por agressões & $-0,02$ & $Y=1,24+4,34$ * ICS & 0,0 \\
\hline
\end{tabular}

ICS: indicador composto de carência social.

* Estatisticamente significativo no nível de 5\%;

** Estatisticamente significativo no nível de 1\%.

transporte, eventos cuja intenção é indeterminada, agressões e lesões autoprovocadas voluntariamente.

Os acidentes de transporte figuraram com maior expressividade dentre as causas externas quanto à associação com a carência social, apresentando correlação positiva de 0,27 . Idosos residentes nos bairros de alta carência social apresentam risco de morte por acidentes de transporte 2,9 vezes maior do que idosos residentes nos bairros do Recife de baixa carência social.

\section{Discussão}

Na paisagem do Recife, a estreita convivência entre ricos e pobres compõe um mosaico de contrastantes condições de habitabilidade que se avizinham em todos os seus bairros e evidenciam processos históricos de desenvolvimento desigual 32 .

A urbanização da cidade do Recife vem produzindo espaços intra-urbanos extremamente desiguais. Esta diferenciação social do espaço não significa apenas desigualdades nas "formas 
de morar". Mais abrangente, ela tem um impacto na qualidade de vida e de saúde dos grupos populacionais residentes em áreas insalubres e desprovidas de serviços de infra-estrutura urbana. As desigualdades em saúde no Recife estão polarizadas nos diferentes estratos de condição de vida. Os estratos de pior e intermediária situação, em geral, apresentam indicadores de saúde menos favoráveis do que o estrato de melhor condição de vida 15,33.

As doenças do aparelho circulatório são as maiores causas de morte entre os idosos recifenses. O segundo grupo de causas de morte em magnitude é representado pelas neoplasias, seguidas das doenças do aparelho digestivo, doenças infecciosas e parasitárias e causas externas. Esta distribuição está de acordo com o padrão nacional, como referem Lima-Costa et al. ${ }^{24}$, no entanto estas mortes não acontecem de forma homogênea em todos os grupos sócio-econômicos.

Huisman et al. 17 referem incremento da mortalidade em idosos europeus com menor nível de escolaridade, em comparação a grupos de maior nível. As doenças cardiovasculares apresentaram maior contribuição para as diferenças de mortalidade. As maiores causas específicas que contribuíram para esta associação foram doença isquêmica do coração, doença pulmonar obstrutiva crônica, outras doenças cardiovasculares e doenças cerebrovasculares. O câncer de pulmão foi mais freqüente entre homens e a pneumonia entre as mulheres.

A mortalidade em idosos por neoplasias malignas de brônquios e pulmões e de mama apresentou correlação negativa com a carência social. Pode-se inferir que com o aumento da carência social, há diminuição dos coeficientes de mortalidade para estas neoplasias.

Boschi et al. 34 realizaram estudo de diferenciais regionais de mortalidade por câncer do Estado do Rio de Janeiro. As autoras referem que a urbanização é um importante fator extrínseco associado à determinação ambiental do câncer. A mortalidade por neoplasias de pulmão, próstata, mama e cólon está associada com a urbanização e alto nível sócio-econômico. Tal informação corrobora os resultados de Cervi et al. ${ }^{35}$. As autoras referem maiores taxas de mortalidade por neoplasias nas regiões Sul e Sudeste, regiões mais desenvolvidas do país, em comparação ao Norte, Nordeste e Centro-Oeste.

As doenças do aparelho circulatório, em especial a pneumonia, apresentaram correlação positiva com a carência social, inferindo-se aumento da mortalidade em grupos menos favorecidos socialmente. Huisman et al. 17 relatam aumento da mortalidade por pneumonia e doença pulmo- nar obstrutiva crônica em idosos europeus com menor nível de escolaridade.

No presente estudo não foi encontrada associação estatisticamente significativa entre a mortalidade por diabetes e a carência social. No entanto, Turrell \& Mathers ${ }^{9}$ relatam o incremento da mortalidade por diabetes na população australiana que reside em áreas de pior condição sócio-ambiental.

A mortalidade por desnutrição em idosos apresentou significativa associação com a carência social. A desnutrição está relacionada a quadros carenciais crônicos ou agudos, é o distúrbio nutricional mais importante em idosos e está associada ao aumento da mortalidade geral, ao aumento da susceptibilidade às infecções e à redução da qualidade de vida. $\mathrm{O}$ problema da mortalidade por desnutrição em idosos incorre em questões importantes sob a ótica social, como a desigualdade sócio-econômica e o papel das políticas públicas 36 .

Apesar do avanço observado na situação de saúde brasileira, representado pelo processo de transição epidemiológica, as doenças infecciosas ainda constituem importante causa de óbito. Ainda mais quando são levadas em consideração as iniqüidades sociais, conferindo ao país um quadro de transição múltipla, com prejuízo para os grupos populacionais mais carentes. As doenças infecciosas são associadas às condições ambientais e de pobreza, quanto maiores as taxas de mortalidade por este grupo de causas, menor é a condição sócio-econômica da população acometida.

Santos \& Noronha 12 referem que a taxa de mortalidade por doenças infecciosas tem relação direta com a proporção de setores subnormais e de domicílios alugados e relação inversa à renda.

Dentre todas as causas de morte em idosos analisadas nesta pesquisa a tuberculose em vias respiratórias apresentou mais forte associação com a carência social. Chaimowicz 37 relata o deslocamento da incidência da tuberculose para a população idosa. O autor refere que o diagnóstico difícil desta patologia na faixa etária de 60 anos e mais pode determinar a elevada mortalidade. Vendramini et al. 38 referem que a tuberculose no idoso é expressa como o recrudescimento de infecção longamente inativa, o idoso está mais predisposto à reinfecção, tanto endógena como exógena. Dentre os eventos antecedentes, identificou-se a moradia nas instituições para idosos como importante fonte de contágio da doença.

Hino et al. 39 consideram a tuberculose uma doença estritamente ligada à desigualdade social. Em estudo realizado no Município de Olinda, Pernambuco, Souza et al. 40 reportaram a alta 
incidência de tuberculose na cidade e a associação estatisticamente significativa com as variáveis, média de moradores por domicílio, existência de famílias com mais de um caso no período e presença de retratamento.

Concomitante ao aumento da carência social, observou-se aumento da mortalidade por causas externas na cidade do Recife. Esta associação é relatada pela literatura. Mackenbach et al. 5 referem aumento do risco de morte por causas externas em homens e mulheres pertencentes a grupos sociais menos favorecidos da Europa. Resultado semelhante é descrito por Turrel \& Mathers 9 na Austrália.

No conjunto das violências, as que mais vitimam os idosos no Brasil são os acidentes de trânsito e transporte, as quedas e os homicídios. Essas três causas específicas representavam $54,1 \%$ do total de óbitos por causas externas entre idosos brasileiros em 198041.

Em estudo realizado no Município de Maringá, Paraná, Mathias et al. 42 analisaram a morbimortalidade por causas externas em idosos residentes na cidade. Os autores referem maior prevalência de quedas e suas conseqüências. A fratura de quadril é uma das conseqüências mais graves. Souza et al. ${ }^{43}$, em estudo sobre os aspectos epidemiológicos dos acidentes de trânsito na população idosa de Maringá, relataram que a maioria das vítimas, $52 \%$, era pedestres, seguidos de condutores e ciclistas.

No Brasil, idosos vítimas fatais de acidentes de trânsito encontram-se predominantemente na condição de pedestre ${ }^{43}$. Na cidade do Recife, a mortalidade de idosos por acidentes de trânsito está associada à maior carência social da população.

Na interpretação dos dados deste estudo ressalta-se que a abordagem da situação de saúde segundo condições de vida ainda apresenta limitações conceituais, metodológicas e técnicas, in- clusive pela dificuldade de operacionalização de variáveis sociais, como refere Silva Jr. ${ }^{44}$. As associações encontradas podem ser mais fortes, em virtude de a agregação espacial utilizada nesta pesquisa ser o bairro. Os bairros do Recife ainda apresentam significativa heterogeneidade social, com áreas de riqueza convivendo com bolsões de pobreza.

Esta pesquisa optou por caracterizar a mortalidade de idosos através do ICS, já utilizado em diversos trabalhos. Há relatos de que os indicadores sintéticos não captam as diversas nuances da realidade social. No entanto, os autores consideram este indicador uma importante ferramenta para caracterização de áreas de risco social, possuindo as qualidades de um bom indicador, referidas por Kerr-Pontes \& Rouquayrol 45, a saber, disponibilidade de dados, simplicidade técnica, uniformidade, sinteticidade e poder discriminatório.

Espera-se que o conhecimento destas associações possa contribuir para a melhoria da qualidade de vida dos idosos recifenses, favorecendo medidas de intervenções setoriais focalizadas para cada grupo populacional.

O crescimento da população de idosos, em números absolutos e relativos é um fenômeno mundial e está ocorrendo em níveis sem precedentes, apenas recentemente as condições de vida da população idosa brasileira passaram a ser alvo de estudos.

A produção científica na temática de condições sócio-econômicas, e sua relação com a situação de saúde na população idosa do Brasil, apresenta-se bastante tímida e no tocante à mortalidade é inexistente. O desenvolvimento de mais estudos nesta área é primordial para a produção de políticas públicas, voltadas ao segmento idoso, que levem em consideração a iniqüidade sócio-econômica tão enraizada na sociedade brasileira. 


\section{Resumo}

Este trabalho objetivou analisar a associação entre carência social e causas de morte da população idosa residente na cidade do Recife, Pernambuco, Brasil, no ano 2000. Realizou-se um estudo ecológico, cuja unidade de análise foi composta por 94 bairros e cinco estratos de carência social. A variável independente consistiu de um indicador composto de carência social, obtido para cada bairro e calculado através da técnica de formação de escores, a partir de variáveis censitárias: abastecimento de água, instalação sanitária, analfabetismo, anos de estudo e renda do chefe de domicílio. As variáveis dependentes foram: coeficiente de mortalidade em maiores de 60 anos e coeficientes específicos em causa básica do óbito. A associação foi calculada através do coeficiente de correlação de Pearson, regressão linear e razão de mortalidade entre os estratos de carência social formados a partir do agrupamento dos bairros por quintil do indicador. Os dados referem correlação positiva estatisticamente significativa da carência social com a mortalidade de idosos ocasionada por pneumonia, desnutrição protéico-calórica, tuberculose, diarréia/gastroenterite e acidentes de transporte e correlação negativa para mortes por neoplasias de brônquios/pulmões e de mama.

Mortalidade; Idoso; Desigualdade Social

\section{Referências}

1. Carvalho JAM, Garcia RA. O envelhecimento da população brasileira: um enfoque demográfico. Cad Saúde Pública 2003; 19:725-33.

2. Teixeira CF. Transição epidemiológica, modelo de atenção à saúde e previdência social no Brasil: problematizando tendências e opções políticas. Ciênc Saúde Coletiva 2004; 9:841-3.

3. Castellanos PL. Perfis de mortalidade, nível de desenvolvimento e iniqüidades sociais na região da Américas. In: Barata RB, organizadora. Equidade e saúde: contribuições da epidemiologia. Rio de Janeiro: Editora Fiocruz/ABRASCO; 1997. p. 137-60.

4. Mackenbach JP, Kunst AE, Cavelaars AEJM, Groenhof F, Geurts JJM. Socioeconomic inequalities in morbidity and mortality in Western Europe. Lancet 1997 349:1655-9.

5. Mackenbach JP, Bos V, Andersen O, Cardano M, Costa G, Harding S. Widening socioeconomic inequalities in mortality in six Western European countries. Int J Epidemiol 2003; 32:830-7.

6. Knesebeck O, Lüschen G, Cockerham WC, Siegrist J. Socioeconomic status and health among the aged in the United States and Germany: A comparative cross-sectional study. Soc Sci Med 2003; 57:1643-52.

7. Nakaya T, Dorling D. Geographical inequalities of mortality by income in two developed island countries: a cross-national comparison of Britain and Japan. Soc Sci Med 2005; 60:2865-75.

\section{Colaboradores}

A proposta do artigo, o levantamento e análise de dados foram realizados por V. L. Silva. A análise estatística foi realizada por J. G. Marino. A discussão dos dados e elaboração do texto foi realizada por V. L. Silva e M. C. C. Leal. A revisão final do texto foi realizada por A. P. O. Marques.

\section{Agradecimentos}

Ao professor Fábio Lessa (in memoriam), pelo importante auxílio na concepção e delineamento deste estudo. Aos professores Sandra Valongueiro e Ricardo Ximenes, pela ajuda na padronização dos indicadores de mortalidade.
8. Khang YH, Lynch JW, Kaplan GA. Health inequalities in Korea: age and sex specific educational differences in the 10 leading causes of death. Int J Epidemiol 2004; 33:299-308.

9. Turrell G, Mathers C. Socioeconomic inequalities in all-cause and specific-cause mortality in Austrália: 1985-1987 and 1995-1997. Int J Epidemiol 2001; 30:231-9

10. Somoza SN, Palladino AC. Condiciones de vida y niveles de salud en la provincia de Formosa. Rev Fac Med Univ Nac Nordeste 2003; 21:11-6.

11. Silva LMV, Paim JS, Costa MCN. Desigualdades na mortalidade, espaço e estratos sociais. Rev Saúde Pública 1999; 33:187-97.

12. Santos SM, Noronha CP. Padrões espaciais de mortalidade e diferenciais sócio-econômicos na cidade do Rio de Janeiro. Cad Saúde Pública 2001; 17:1099-110.

13. Neri M, Soares W. Desigualdade social e saúde no Brasil. Cad Saúde Pública 2002; 18 Suppl:S77-87.

14. Guimarães MJB, Marques NM, Melo Filho DA, Szwarcwald CL. Condição de vida e mortalidade infantil: diferenciais intra-urbanos no Recife, Pernambuco, Brasil. Cad Saúde Pública 2003; 19:1413-24. 
15. Silva ICA. Contribuição à vigilância da saúde na cidade do Recife: Estudo das desigualdades de condição de vida e de saúde - 1996-2001 [Dissertação de Mestrado]. Recife: Universidade Federal de Pernambuco; 2003.

16. Huisman M, Knust AE, Andersen O, Bopp M, Borgan JK, Borrell C, et al. Socioeconomic inequalities in mortality among elderly people in 11 European populations. J Epidemiol Community Health 2004; 58:468-75

17. Huisman M, Knust AE, Bopp M, Borgan JK, Borrell C, Costa G, et al. Educational inequalities in cause-specific mortality in middle-aged and older men and women in eight Western European populations. Lancet 2005; 365:493-500.

18. Noronha KVMS, Andrade MV. Desigualdades sociais em saúde e na utilização dos serviços de saúde entre idosos na América Latina. Rev Panam Salud Pública 2005; 17:410-8.

19. Lima-Costa MF, Barreto S, Giatti L. A situação socioeconômica afeta igualmente a saúde de idosos e adultos mais jovens no Brasil? Um estudo utilizando dados da Pesquisa Nacional por Amostras de Domicílios, PNAD/98. Ciênc Saúde Coletiva 2002; 7:813-24

20. Lima-Costa MF, Barrteo S, Giatti L, Uchôa E. Desigualdade social e saúde entre idosos brasileiros: um estudo baseado na Pesquisa Nacional por amostra de domicílios. Cad Saúde Pública 2003; 19:745-57.

21. Drumond Jr. M, Barros MBA. Desigualdades socioespaciais na mortalidade do adulto no município de São Paulo. Rev Bras Epidemiol 1999; 2:34-49.

22. Barros BAB, César CLG, Carandina L, Torre GD. Desigualdades sociais na prevalência de doenças crônicas no Brasil, PNAD-2003. Ciênc Saúde Coletiva 2006; 11:911-26.

23. Paes-Souza R. Diferenciais intra-urbanos de mortalidade em Belo Horizonte, Minas Gerais, Brasil, 1994: revisitando o debate sobre transições demográfica e epidemiológica. Cad Saúde Pública 2002; 18:1411-21.

24. Caramano AA. Envelhecimento da população brasileira: uma contribuição demográfica. In: Freitas EV, organizadora. Tratado de geriatria e gerontologia. Rio de Janeiro: Editora Guanabara Koogan; 2002. p. 58-71.

25. Lima-Costa MF, Peixoto SV, Giatti L. Tendências de mortalidade entre idosos brasileiros (1980-2000). Epidemiol Serv Saúde 2004; 13:217-28.

26. Bonfim C. Filariose Bancroftiana: a representação espacial das desigualdades sociais [Dissertação de Mestrado]. Recife: Centro de Pesquisas Aggeu Magalhães, Fundação Oswaldo Cruz; 2002.

27. Souza WV. O uso de informações de indicadores de situação coletiva de risco para a ocorrência de tuberculose em Olinda - PE [Dissertação de Mestrado]. Recife: Centro de Pesquisas Aggeu Magalhães, Fundação Oswaldo Cruz; 1998.

28. Ximenes RAA, Martelli CMT, Souza WV, Lapa TM, Albuquerque MFM, Andrade ALSS, et al. Vigilância de doenças endêmicas em áreas urbanas: a interface entre mapas de setores censitários e indicadores de mortalidade. Cad Saúde Pública 1999; $15: 53-62$.
29. Kirkwood BR. Essentials of medical statistics. Oxford: Blackwell Science; 1988.

30. Organização Mundial da Saúde. Classificação estatística internacional de doenças e problemas relacionados à saúde, 10a revisão. v. 1. São Paulo: Centro Colaborador da OMS para a Classificação de Doenças em Português; 1995.

31. Ministério da Saúde. Saúde Brasil 2005: uma análise da situação de saúde. Brasília: Ministério da Saúde; 2005.

32. Miranda L. Desenvolvimento humano e habitação no Recife. Atlas do desenvolvimento humano no Recife [CD-ROM]. Recife: Secretaria de Planejamento Participativo, Obras e Desenvolvimento Urbano e Ambiental; 2005.

33. Albuquerque MFPM. Urbanização, favelas e endemias: a produção da filariose no Recife, Brasil. Cad Saúde Pública 1993: 9:487-97.

34. Boschi C, Coleman MP, Castilho EA. Diferenciais regionais de mortalidade por câncer no Estado do Rio de Janeiro, Brasil, 1979-1981. Rev Saúde Pública $1991 ; 25: 267-75$.

35. Cervi A, Hermsdorff HHM, Ribeiro RCL. Tendência de mortalidade por doenças neoplásicas em 10 capitais brasileiras, de 1980 a 2000. Rev Bras Epidemiol 2005; 8:407-18.

36. Otero UB, Rozenfeld S, Gadelha AJ. Óbitos por desnutrição em idosos, São Paulo e Rio de Janeiro. Análise de séries temporais, 1980-1996. Rev Bras Epidemiol 2001; 4:191-205.

37. Chaimowicz F. Age transition of tuberculosis incidence and mortality in Brazil. Rev Saúde Pública 2001; 35:81-7.

38. Vendramini SHF, Villa TCS, Gonzales RIC, Monroe AA. Tuberculose no idoso: análise do conceito. Rev Latinoam Enferm 2003; 11:96-103.

39. Hino P, Santos CB, Villa TCS. Evolução espaçotemporal dos casos de tuberculose em Ribeirão Preto (SP), nos anos de 1998 a 2002. J Bras Pneumol 2005; 31:523-27.

40. Souza WV, Albuquerque MFM, Barcellos CC, Ximenes RAA, Carvalho MS. Tuberculose no Brasil: construção de um sistema de vigilância de base territorial. Rev Saúde Pública 2005; 39:82-9.

41. Minayo MCS. Violência contra idosos: relevância para um velho problema. Cad Saúde Pública 2003; 19:783-91.

42. Mathias TAF, Jorge MHM, Andrade OG. Morbimortalidade por causas externas na população idosa residente em município da região Sul do Brasil. Rev Latinoam Enferm 2006; 14:17-24.

43. Souza RKT, Soares DFPP, Mathias TAF, Andrade OG, Santana RG. Idosos vítimas de acidentes de trânsito: aspectos epidemiológicos e impacto na sua vida cotidiana. Acta Sci 2003; 25:19-25.

44. Silva Jr. JB. Diferenciais intra-urbanos de saúde em Olinda/PE [Dissertação de Mestrado]. Campinas: Universidade Estadual de Campinas; 1995.

45. Kerr-Pontes LS, Rouquayrol MZ. Medida da saúde coletiva. In: Rouquayrol MZ, Almeida Filho N, organizadores. Epidemiologia e saúde. Rio de Janeiro: Editora Medsi; 2003. p. 37-82.

Recebido em 02/Abr/2007

Versão final reapresentada em 10/Set/2007 Aprovado em 01/Out/2007 